\title{
Harmonically confined particles with long-range repulsive interactions
}

\author{
S. Agarwal, ${ }^{1,2}$ A. Dhar, ${ }^{1}$ M. Kulkarni, ${ }^{1}$ A. Kundu,${ }^{1}$ S. N. Majumdar, ${ }^{3}$ D. Mukamel,${ }^{4}$ and G. Schehr ${ }^{3}$ \\ ${ }^{1}$ International Centre for Theoretical Sciences, Tata Institute of Fundamental Research, Bengaluru - 560089, India \\ ${ }^{2}$ Birla Institute of Technology and Science, Pilani - 333031, India \\ ${ }^{3}$ LPTMS, CNRS, Univ. Paris-Sud, Universite Paris-Saclay, 91405 Orsay, France \\ ${ }^{4}$ Department of Physics of Complex Systems, Weizmann Institute of Science, Rehovot 7610001, Israel
}

(Dated: July 23, 2019)

\begin{abstract}
We study an interacting system of $N$ classical particles on a line at thermal equilibrium. The particles are confined by a harmonic trap and repelling each other via pairwise interaction potential that behaves as a power law $\propto \sum_{i \neq j}^{N}\left|x_{i}-x_{j}\right|^{-k}$ (with $k>-2$ ) of their mutual distance. This is a generalization of the well known cases of the one component plasma $(k=-1)$, Dyson's log-gas $\left(k \rightarrow 0^{+}\right)$, and the Calogero-Moser model $(k=2)$. Due to the competition between harmonic confinement and pairwise repulsion, the particles spread over a finite region of space for all $k>-2$. We compute exactly the average density profile for large $N$ for all $k>-2$ and show that while it is independent of temperature for sufficiently low temperature, it has a rich and nontrivial dependence on $k$ with distinct behavior for $-2<k<1, k>1$ and $k=1$.
\end{abstract}

PACS numbers:

Introduction: A gas of $N$ classical particles, confined by a harmonic potential on a line and interacting with each other via pairwise repulsion, constitutes one of the simplest interacting particle systems that have been well studied in the past. It has seen a recent revival in the wake of the physics of cold atoms. When the pairwise repulsive interaction decays as a power-law of the distance between the particles, the energy of the so called Riesz gas $^{1}$ is given by

$$
E\left(\left\{x_{i}\right\}\right)=\frac{1}{2} \sum_{i=1}^{N} x_{i}^{2}+\frac{J \operatorname{sgn}(k)}{2} \sum_{i \neq j} \frac{1}{\left|x_{i}-x_{j}\right|^{k}}
$$

where $J>0$ and $\left\{x_{i}\right\}(i=1,2, \ldots, N)$ denote the positions of the particles on the line. The index $k>-2$ characterizes the strength of the pairwise interaction and $\operatorname{sgn}(k)$ in the prefactor ensures a repulsive interaction. For $k<-2$, the quadratic potential is not strong enough to counter the strong repulsion and confine the particles. Consequently the particles fly off to $\pm \infty$ and thus the case $k<-2$ is not physically interesting. Given the energy in (1), the joint probability distribution function (PDF) of the particles' positions is given by the Boltzmann weight, $P\left(x_{1}, \cdots, x_{N}\right)=\frac{1}{Z_{N}(\beta)} e^{-\beta E\left[\left\{x_{i}\right\}\right]}$, where $\beta$ is the inverse temperature $\left(\mathrm{k}_{\mathrm{B}}=1\right)$ and $Z_{N}(\beta)=$ $\int \prod_{i=1}^{N} d x_{i} e^{-\beta E\left[\left\{x_{i}\right\}\right]}$ is the normalizing partition function. The harmonic potential tries to confine the particles near the center of the trap, while the repulsive interaction tries to push them apart. As a result of the competition between the two terms, it turns out that the particles get confined to a finite region of space for large $N$, with a space-dependent average macroscopic density, $\left\langle\rho_{N}(x)\right\rangle=N^{-1} \sum_{i=1}^{N}\left\langle\delta\left(x-x_{i}\right)\right\rangle$ (normalized to unity), where $\langle\ldots\rangle$ denotes an average with respect to the Boltzmann weight. A basic natural question is: what is the configuration of $x_{i}$ 's that minimizes the energy in (1) for large $N$ and what is the density profile in the ground state? This is a classic and important optimization prob- lem both in physics (see below) and in mathematics (see e.g. Refs. 2-4) whose solution, for generic $k>-2$, is hitherto unknown. A related question is: how does the average density profile depend on the inverse temperature $\beta$ ?

This problem is of great general interest as there are varied physical systems that correspond to special values of $k$. We start with $k=-1$ where the interaction is linearly repulsive with distance. This is the well known one dimensional one-component plasma $(1 d \mathrm{OCP})^{5}$, consisting of oppositely charged particles with pairwise Coulomb interaction (linear in 1d) and overall charge neutrality ${ }^{6-10}$. Integrating out the positions of the negative charges gives rise to an effective quadratic confinement for the positive charges and the effective energy of the $N$ positive charges with coordinates $\left\{x_{i}\right\}$ is precisely given by (1) with $k=-1$. In this case, the energy can be easily minimized by ordering the positions of the particles leading to an equispaced configuration ${ }^{6-10}$. Moreover, for large $N$, the average density profile $\left\langle\rho_{N}(x)\right\rangle$ turns out to be independent of $\beta$ and approaches a scaling form $\left\langle\rho_{N}(x)\right\rangle \rightarrow(1 / N) \tilde{\rho}_{\mathrm{OCP}}(x / N)$, where the scaled density $\tilde{\rho}_{\mathrm{OCP}}(y)=1 /(2 J)$ is uniform over the interval $[-J,+J]$ and vanishes outside ${ }^{3,6-11}$.

The second and perhaps the most well studied example corresponds to the limit $k \rightarrow 0^{+}$, where we replace $\operatorname{sgn}(k)$ in Eq. (1) by +1 , use $\left|x_{i}-x_{j}\right|^{(-k)} \approx 1-k \log \left|x_{i}-x_{j}\right|$ and set $J=1 / k$. The energy in (1) then reduces, up to an overall additive constant, to

$$
E\left[\left\{x_{i}\right\}\right]=\frac{1}{2} \sum_{i=1}^{N} x_{i}^{2}-\frac{1}{2} \sum_{i \neq j} \ln \left|x_{i}-x_{j}\right| .
$$

This is the celebrated log-gas of Dyson ${ }^{12}$. For the special values of $\beta=1,2$ and 4 , the Boltzmann weight of the log-gas $P\left(\left\{x_{i}\right\}\right)=\frac{1}{Z_{N}(\beta)} \exp \left[-\beta E\left[\left\{x_{i}\right\}\right]\right]$ can be identified with the joint distribution of $N$ real eigenvalues of an $N \times N$ matrix belonging to the Gaussian ensembles of the 
random matrix theory (RMT): respectively Gaussian Orthogonal Ensemble (GOE), Gaussian Unitary Ensemble (GUE) and Gaussian Symplectic Ensemble (GSE) ${ }^{5,13}$. Gaussian ensembles are the cornerstones of RMT with myriads of applications, ranging from nuclear physics, mesoscopic transport, quantum chaos, number theory all the way to finance and big-data science ${ }^{5,13-15}$. The loggas with arbitrary $\beta>0$ also appears in RMT as the joint PDF of the eigenvalues of the so called DumitriuEdelman $\beta$-ensemble of tridiagonal random matrices ${ }^{16}$. The average density for large $N$ converges to the scaling form, independently of $\beta$,

$$
\left\langle\rho_{N}(x)\right\rangle \approx \frac{1}{\sqrt{N}} \tilde{\rho}_{\mathrm{sc}}\left(\frac{x}{\sqrt{N}}\right), \tilde{\rho}_{\mathrm{sc}}(y)=\frac{1}{\pi} \sqrt{2-y^{2}} .
$$

Thus the scaled density is supported over $[-\sqrt{2},+\sqrt{2}]$ and is known as the celebrated Wigner semi-circular $\mathrm{law}^{17}$. This central result of RMT has been instrumental in understanding the global properties in a variety of systems including growth models in $1+1$ dimensions belonging to the KPZ universality class ${ }^{18}$, non-interacting trapped fermions ${ }^{19-21}$ where the Wigner semi-circle can also be obtained from the so-called local density approximation $(\mathrm{LDA})^{22}$, non-intersecting Brownian motions $^{23-25}$, graph theory and communication networks ${ }^{14}$.

The third physical example corresponds to $k=2$, i.e., with inverse-square repulsion. For $k=2,(1)$ is the celebrated Calogero-Moser model ${ }^{26-29}$ which is integrable and is ubiquitous in diverse fields ${ }^{30}$. Curiously, it turns out that for any finite $N$, in the minimum energy configuration of both the log-gas $\left(k \rightarrow 0^{+}\right)$and the CalogeroMoser model $(k=2)$, the particle positions $x_{i}$ 's coincide exactly ${ }^{31,32}$ with the $N$ zeros of the Hermite polynomial of degree $N$. Consequently, for large $N$, the scaled average density for $k=2$ also approaches the Wigner semicircular law in Eq. (3) and as in the log-gas case, the semicircular law is independent of $\beta$. This is rather strange: even though the long-range repulsive interaction in the log-gas is much stronger than that of the inverse-square gas, the average density profile is identical in the two cases. This raises a very interesting and natural question: How does the shape of the average scaled density for large $N$ vary as one tunes the parameter $k$ ? The appearance of the semi-circular law both for $k \rightarrow 0^{+}$ and $k=2$ suggests an intriguing possibility of a 'nonmonotonic' dependence of the shape of the average density as one tunes up $k$. This question on the dependence of the average density on $k$ also has practical implications in a number of physical contexts. For example, in a typical cold atom experimental set-up, a quadratic confining potential is natural due to the usage of optical laser traps. In addition, it is possible to induce long-range power-law repulsive interactions between such atoms. For instance, charged particles interacting via the $3 d$ Coulomb repulsion, but confined to a line by highly anisotropic optical trap, would correspond to $k=1$. Similarly, $k=3$ describes a dipolar gas confined to $1 d^{33-35}$. For $k \rightarrow \infty$, the Riesz gas reduces to a truely short-ranged repulsive gas similar to the harmonically confined screened Coulomb or Yukawa-gas studied in Refs. 11,36. In addition, other values of $k$ have either been realized in experimental setups or could potentially be realized ${ }^{37}$.

In this Letter, we address this interesting question of the dependence of the average density on $k$ and obtain exact results for large $N$. We show that for large $N$ the average density is independent of $\beta$ for all $k$ (for sufficiently large $\beta)^{38}$, but has a rich and nontrivial dependence on $k$. On general grounds the average density is expected to have a scaling form $\left\langle\rho_{N}(x)\right\rangle \approx N^{-\alpha_{k}} \tilde{\rho}_{k}\left(x / N^{\alpha_{k}}\right)$ for large $N$, where $N^{\alpha_{k}}$ corresponds to the typical scale of the position of the particles in the trap. Indeed, we do find this behavior, but with a twist. We show that there is a drastic change of behavior of the exponent $\alpha_{k}$ as well as the scaling function $\tilde{\rho}_{k}(y)$ at $k=1$. For the exponent, we get

$$
\alpha_{k}=\left\{\begin{array}{cc}
\frac{1}{k+2}, & -2<k<1 \\
\frac{k}{k+2}, & k>1 .
\end{array}\right.
$$

The scaling function $\tilde{\rho}_{k}(y)$ has a support over $\left[-\ell_{k} / 2,+\ell_{k} / 2\right]$ and can be expressed as $\tilde{\rho}_{k}(y)=$ $\ell_{k}^{-1} F_{k}\left(y / \ell_{k}\right)$ where $F_{k}(z)$ is given by

$$
F_{k}(z)=\frac{1}{B\left(\gamma_{k}+1, \gamma_{k}+1\right)}\left(\frac{1}{4}-z^{2}\right)^{\gamma_{k}} .
$$

with $-1 / 2 \leq z \leq 1 / 2$ and $B(a, b)$ is the standard Beta function. Thus the density either diverges or vanishes at the two scaled edges $z= \pm 1 / 2$ with an exponent $\gamma_{k}$ which also exhibits a change of behavior at $k=1$, namely

$$
\gamma_{k}= \begin{cases}\frac{k+1}{2}, & -2<k<1 \\ \frac{1}{k}, & k>1 .\end{cases}
$$

The support length $\ell_{k}$ is non-universal and depends explicitly on $k$ and the coupling strength $J$ (for the exact expressions of $\ell_{k}$, see Eqs. (36) and (65) of Supp. Mat. $\left.{ }^{45}\right)$. The scaling function $F_{k}(z)$ depends only on $k$, and is independent of $\beta$ and $J$. The case $k=1$ is marginal with additional logarithmic corrections (we discuss this later). We show that this change of behavior at $k=1$ can be traced back to the fact that, for $k<1$, the large distance behavior of the interaction term controls the large $N$ behavior of the density. In contrast, for $k>1$, the limiting density is determined by the short distance behavior of the interaction term. This gives rise to an effective field-theory that is fundamentally different for $k<1$ and $k>1$. Thus $k \rightarrow 0^{+}$(log-gas) and $k=2$ (inverse-square gas) share the same average density profile, but the physics is rather different in the two cases. For $k \rightarrow-1$, we recover the flat density of the $1 d \mathrm{OCP}$. Also, in the limit $k \rightarrow \infty$ we again get a flat density, consistent with the results for the $1 d$ harmonically confined Yukawa gas ${ }^{11,36}$. We also performed Monte-Carlo (MC) simulations for several values of $k$, finding excellent agreement with our analytical predictions (see Fig. 1). 
Regime 1: $-2<k<1$ : Assuming both terms in the energy (1) are of the same order for large $N$, the energy scale can be estimated as follows. Let the typical position of a particle scales as $x_{\mathrm{typ}} \sim N^{\alpha_{k}}$ for large $N$, where $\alpha_{k}$ is to be determined. Then the first term in (1) scales as $\sim N^{2 \alpha_{k}+1}$, while the second term (where the double sum contains typically $N(N-1) \approx N^{2}$ terms) scales as $\sim J N^{2-k \alpha_{k}}$. Demanding they are of the same order fixes the exponent $\alpha_{k}=\frac{1}{k+2}$ (see the first line in Eq. (4)). Hence the total energy scales as $E \sim N^{2 \alpha_{k}+1} \sim N^{\frac{4+k}{2+k}}$ in this regime. To find the configuration that dominates the partition function $Z_{N}(\beta)$ for large $N$, we generalise the method used for the log-gas $\left(k \rightarrow 0^{+} \text {limit }\right)^{12,48-50}$. It turns out to be convenient to express the coarse grained energy in terms of a macroscopic density $\rho_{N}(x)$ and use the relation $\sum_{i=1}^{N} f\left(x_{i}\right) \approx N \int f(x) \rho_{N}(x) d x$, valid for any smooth function $f(x)$. Next, we rescale $x=N^{\alpha_{k}} y$ with $y \sim O(1)$. Under this rescaling, the density transforms as $\rho_{N}(x) \approx N^{-\alpha_{k}} \tilde{\rho}_{k}\left(y=x N^{-\alpha_{k}}\right)$, where we assume $\tilde{\rho}_{k}(y)$ is smooth and normalizable, $\int \tilde{\rho}_{k}(y) d y=1$. Consequently, the coarse grained partition function for large $N$ can be expressed as a functional integral over the density field $\tilde{\rho}_{k}(y)$ (for details see Supp. Mat. ${ }^{45}$ )

$$
Z_{N}(\beta) \sim \int d \mu \int \mathcal{D}\left[\tilde{\rho}_{k}\right] \exp \left(-\beta N^{\frac{4+k}{2+k}} \Sigma\left[\tilde{\rho}_{k}(y)\right]\right)
$$

where the action $\Sigma\left[\tilde{\rho}_{k}(y)\right]$ is given by (see also Ref. 51 for a rigorous proof in the cases $0<k<1$ )

$$
\begin{aligned}
\Sigma\left[\tilde{\rho}_{k}(y)\right] & =\frac{J \operatorname{sgn}(k)}{2} \int d y \int d y^{\prime} \frac{\tilde{\rho}_{k}(y) \tilde{\rho}_{k}\left(y^{\prime}\right)}{\left|y-y^{\prime}\right|^{k}} \\
& +\frac{1}{2} \int d y y^{2} \tilde{\rho}_{k}(y)-\mu\left(\int d y \tilde{\rho}_{k}(y)-1\right) .
\end{aligned}
$$

Here $\mu$ is the Lagrange multiplier that enforces the constraint $\int \tilde{\rho}_{k}(y) d y=1$. Note that in the integrand of Eq. (7), we have only kept the leading order contributions to the energy. Both the entropy term (generated in going from microscopic configurations to the macroscopic density) as well as the short distance behavior of the interaction energy have been neglected, as they are of lower order in $N$ for $-2<k<1$. This is valid as long as $\beta \gg N^{-2 \alpha_{k}}$ where $\alpha_{k}=1 /(k+2)^{38,45}$. Thus the effective action $\Sigma\left[\tilde{\rho}_{k}(y)\right]$ is manifestly non-local reflecting the long-range nature of the repulsive interaction. We will see later that this non-locality manifests only for $-2<k<1$. For large $N$, the partition function in Eq. (7) can then be evaluated by the saddle point method. Minimizing the action $\Sigma\left[\tilde{\rho}_{k}(y)\right]$ in (8) with respect (w.r.t.) to $\tilde{\rho}_{k}(y)$ gives the saddle point equation for the optimal density

$$
\frac{y^{2}}{2}+J \operatorname{sgn}(k) \int d y^{\prime} \frac{\tilde{\rho}_{k}\left(y^{\prime}\right)}{\left|y^{\prime}-y\right|^{k}}=\mu .
$$

This equation is valid over the support of $\tilde{\rho}_{k}(y)$. The density is clearly symmetric in $y$, hence the support is over $\left[-\ell_{k} / 2, \ell_{k} / 2\right]$ where $\ell_{k}$ is fixed using the normalization $\int_{-\ell_{k} / 2}^{\ell_{k} / 2} \rho_{k}(y) d y=1$. Taking a further derivative of

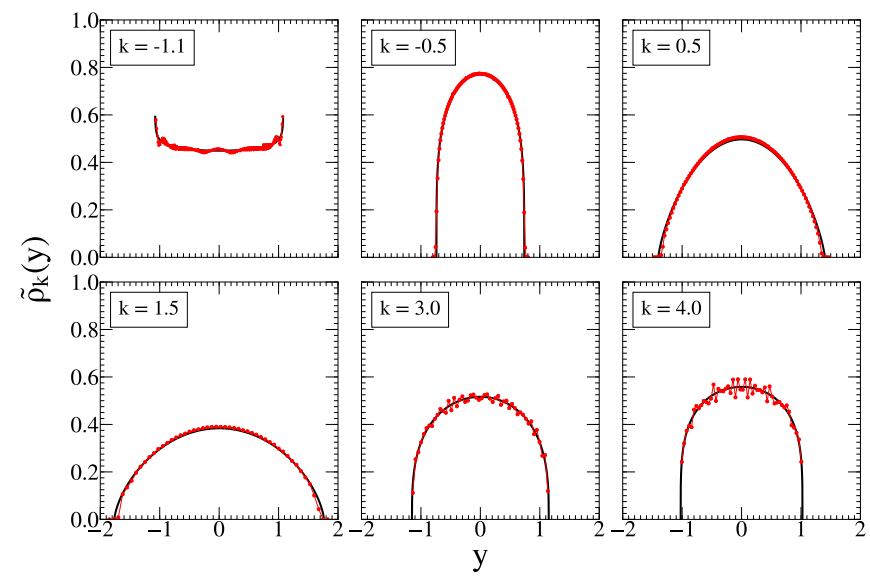

FIG. 1: The numerical (MC) average scaled density $\tilde{\rho}_{k}(y)$ vs $y$ (red dots) for different values of $k$ : for $-2<k<1$ in the top three panels and for $k>1$ in the bottom three panels (where $N=200$ and $\beta=2$ ). The numerical curves are compared to analytical predictions (black) with excellent agreement. Oscillations are somewhat prominent at higher $|k|$ due to the finite $N$ effects. The ensemble average is over $2 \times 10^{8}$ MC samples.

(9) w.r.t. $y$ leads to a singular integral equation

$$
P V \int_{-\ell_{k} / 2}^{\ell_{k} / 2} \frac{\operatorname{sgn}\left(y^{\prime}-y\right)}{\left|y-y^{\prime}\right|^{k+1}} \tilde{\rho}_{k}\left(y^{\prime}\right) d y^{\prime}=-\frac{y}{J|k|}, k \neq 0
$$

where $P V$ denotes the principal value which needs to be taken only for $k>0$. Also, for $k \rightarrow 0, J$ has to be rescaled such that $J|k|=1$. Solving this singular integral equation poses the main technical challenge. Fortunately, it turns out that for $-2<k<1$, this equation can be transformed into the well studied Sonin form ${ }^{52-58}$, and can subsequently be inverted to obtain $\tilde{\rho}_{k}(y)$ explicitly $^{45}$. We then obtain the exact saddle point density $\tilde{\rho}_{k}(y)=\ell_{k}^{-1} F_{k}\left(y / \ell_{k}\right)$ where $\ell_{k}$ is given in Supp. Mat. ${ }^{45}$ and $F_{k}(z)$ is given in Eq. (5) with $\gamma_{k}=(k+1) / 2$. Note that the Sonin inversion formula also indicates that there is no physical solution (saddle point density) for $k>1$. Hence, this solution is valid only in the range $-2<k<1$. Furthermore, since $\beta$ appears only in the factor $\beta N^{(4+k) /(2+k)}$ outside the action $\Sigma\left[\tilde{\rho}_{k}(y)\right]$ in $(7)$, it is clear that the saddle point density is independent of $\beta$ : large $N$ is equivalent to large $\beta$. In addition, the average density $\left\langle\rho_{N}(x)\right\rangle$, for large $N$, clearly coincides with the saddle point density as the average over all possible densities is dominated by the saddle point. In Fig. 1 upper panels, we compare our theoretical predictions with $\mathrm{MC}$ simulations for three representative values of $k$ in the range $-2<k<1$ and find excellent agreement. Note that in the range $-2<k<-1$ the density diverges at the edges $\pm l_{k} / 2$, while for $-1<k<1$ the density vanishes at the edges. Exactly at $k=-1$, the density is flat, consistent with the $1 d \mathrm{OCP}$ result.

Regime 2: $k \geq 1$ : It turns out that, for $k>1$, the interaction term in (1) containing the double sum is dom- 
inated by particles which are very close to each other, i.e., almost nearest neighbours. As a result, the short distance properties of the interaction term plays a more dominant role compared with its long distance behavior. This leads to an effective field theory which is local in the density and is much simpler. To compute the effective coarse grained energy for large $N$, we then take a different path than the $k<1$ case (for details see Supp. Mat. $\left.{ }^{45}\right)$. First, it is convenient to order the particle positions so that $x_{i}$ increases with the label $i$ (this is fine since the energy (1) is invariant under permutation of labels). We then replace the discrete particle label $i$ by a continuous coordinate and the position $x_{i}$ is approximated by a smooth continuous function $x(i)$. Next, we approximate $x_{i}-x_{j} \approx(i-j) x^{\prime}(i)$ where $x^{\prime}(i)=d x(i) / d i$ and we have kept only the first term in the Taylor expansion anticipating that it captures the leading short distance behavior. Our next step is to express $x^{\prime}(i)$ in terms of the local smooth macroscopic density $\rho_{N}(x)$ (normalized to unity). In fact, the local slope of the smooth function $x(i)$, i.e., $x^{\prime}(i)>0$ is simply the inverse of the number density $N \rho_{N}(x)$, i.e., $x^{\prime}(i)=1 /\left(N \rho_{N}(x)\right)$. Thus the double sum in (1) can be approximated, to leading order for large $N$, by $\sum_{i \neq j}\left|x_{i}-x_{j}\right|^{-k}=\sum_{i \neq j}|i-j|^{-k}\left[N \rho_{N}\left(x_{i}\right)\right]^{k}$. The sum over $j$, for fixed $i$, is convergent for all $k>1$ and simply gives a factor $2 \zeta(k)$, where $\zeta(k)=\sum_{n=1}^{\infty} n^{-k}$ is the Riemann zeta function. Furthermore, the sum over $i$ can be replaced by an integral using the relation $\sum_{i=1}^{N} f\left(x_{i}\right) \approx N \int f(x) \rho_{N}(x) d x$ mentioned before. Using this relation in both terms of (1) leads to a coarse grained energy $\mathcal{E} \equiv \mathcal{E}\left[\rho_{N}(x)\right]^{45}$

$$
\mathcal{E} \approx \frac{N}{2} \int d x x^{2} \rho_{N}(x)+J \zeta(k) N^{k+1} \int d x\left[\rho_{N}(x)\right]^{k+1},
$$

which is completely local in the density $\rho_{N}(x)$, unlike (8) for $-2<k<1$ that involved densities at two spaceseparated points. We then rescale $x \rightarrow x N^{-\alpha_{k}}$ and write $\rho_{N}(x)=N^{-\alpha_{k}} \tilde{\rho}_{k}\left(y=x N^{-\alpha_{k}}\right)$. It is easy to see that for both terms in (11) to be of the same order in $N$ for large $N$, we need to choose $\alpha_{k}=k /(k+2)$, as stated in the second line of (4). Hence, the total energy scales as $E \sim N^{\frac{3 k+2}{k+2}}$ for large $N$. The coarse grained partition function $Z_{N}(\beta)$ can then be written as

$$
Z_{N}(\beta) \sim \int d \mu \int \mathcal{D}\left[\tilde{\rho}_{k}\right] \exp \left(-\beta N^{\frac{3 k+2}{k+2}} \Sigma\left[\tilde{\rho}_{k}(y)\right]\right)
$$

where the action $\Sigma\left[\tilde{\rho}_{k}(y)\right]$ is given by

$$
\begin{aligned}
\Sigma\left[\tilde{\rho}_{k}(y)\right] & =\frac{1}{2} \int d y y^{2} \tilde{\rho}_{k}(y)+J \zeta(k) \int d y\left[\tilde{\rho}_{k}(y)\right]^{k+1} \\
& -\mu\left(\int d y \tilde{\rho}_{k}(y)-1\right)
\end{aligned}
$$

with $\mu$ again denoting the Lagrange multiplier enforcing the normalization of the density. Note that we have kept the leading order contribution for large $N$ in the integrand in (12) and again neglected the entropy as well as subdominant singular terms which are of lower order in $N$. For large $N$, the integral (12) can again be evaluated by the saddle point method. Minimizing the action gives the saddle point equation

$$
\frac{1}{2} y^{2}+J \zeta(k)(k+1)\left[\tilde{\rho}_{k}(y)\right]^{k}=\mu .
$$

Trivially solving this equation gives, $\tilde{\rho}_{k}(y)=$ $\left(1 / \ell_{k}\right) F_{k}\left(y / \ell_{k}\right)$, with support over $\left[-\ell_{k} / 2, \ell_{k} / 2\right]$ where $\ell_{k}=2 \sqrt{2 \mu}$ is fixed from the normalization and is given explicitly in Supp. Mat. ${ }^{45}$. The scaling function $F_{k}(z)$ is then of the form in (5) with the exponent $\gamma_{k}=1 / k$. The saddle point density coincides with the average density $\left\langle\rho_{N}(x)\right\rangle$ for large $N$. In addition, since $\beta$ appears only in the combination $\beta N^{(3 k+2) /(k+2)}$ outside the action in (12), clearly the saddle point density and hence the average density $\left\langle\rho_{N}(x)\right\rangle$ is independent of $\beta$ for large $N$, as long as $\beta \gg N^{-2 \alpha_{k}}$ where $\alpha_{k}=k /(k+2)^{38,45}$. This analytical prediction is then verified in $\mathrm{MC}$ simulations (see the bottom panels in Fig. 1).

The marginal case $k=1$ lies at the borderline between Regime 1 and Regime 2. In this case, one would expect logarithmic corrections. Indeed, we find ${ }^{45}$ that the average density approaches a scaling form, $\left\langle\rho_{N}(x)\right\rangle \sim$ $L_{N}^{-1} \tilde{\rho}_{1}\left(x / L_{N}\right)$, where the typical position of a particle scales as $L_{N} \approx(N \ln N)^{1 / 3}$ for large $N$. The scaling function $\tilde{\rho}_{1}(y)$ is supported over $\left[-\ell_{1} / 2, \ell_{1} / 2\right]$ with $\ell_{1}=2 \sqrt{2 \mu}$ and is given by

$$
\tilde{\rho}_{1}(y)=\frac{1}{4 J}\left(2 \mu-y^{2}\right), \quad \mu=\frac{1}{2}(3 J)^{2 / 3} .
$$

This can be also cast in the scaling form $\tilde{\rho}_{1}(y)=$ $\left(1 / \ell_{1}\right) F_{1}\left(y / \ell_{1}\right)$ where $F_{1}(z)$ is given in $(5)$ with $\gamma_{1}=1$. Numerical simulations are in good agreement with our analytical prediction, as shown in the Supp. Mat. ${ }^{45}$.

Conclusions: In this Letter, we have computed analytically the average density profile of a classical gas of harmonically confined particles that repel each other with the repulsive interaction behaving as a power-law with exponent $-k$ of the distance between any pair of particles. Our result generalizes in a nontrivial way, to arbitrary $k>-2$, the three famous classical examples: the $1 d \mathrm{OCP}(k=-1)$, the Dyson's log-gas in $\operatorname{RMT}\left(k \rightarrow 0^{+}\right)$ and the Calogero-Moser model $(k=2)$. We have shown that the underlying effective field theory that determines the average density profile for large $N$ is governed by fundamentally different physics for $-2<k<1$ and $k>1$. In the former case, the large distance behavior of the interaction potential dominates, while the latter case is governed by its short distance behavior. It would be interesting to study other observables beyond the average density for general $k>-2$. For instance, for the loggas $\left(k \rightarrow 0^{+}\right)$the position of the rightmost particle $x_{\max }$ (the largest eigenvalue of a random matrix), centered and scaled, is known to converge to the celebrated TracyWidom distribution ${ }^{59}$. The corresponding extreme value distribution for $k=-1$ has recently been computed exactly $^{9,10}$ and the case $k=2$ has been recently computed 
numerically ${ }^{32}$. It would be interesting to compute the limiting distribution of $x_{\max }$ for generic $k>-2$. Finally, it would be interesting to see if our predictions for the average density can be measured in cold atom experiments. From that perspective, it would be nice to extend our results for the density profile to higher dimensions.

Note added in proof: After submission of the work, we came to know from O. Zeitouni that the case $k>1$ was also studied recently in the mathematics literature ${ }^{60}$.

Acknowledgments: We would like to thank T. Leblé, E. Saff and S. Serfaty for pointing out useful references and O. Zeitouni for stimulating discussions. MK would like to acknowledge support from the project 60041 of the Indo-French Centre for the Promotion of Advanced Research (IFCPAR), the Ramanujan Fellowship SB/S2/RJN-114/2016 and the SERB Early Career Research Award ECR/2018/002085 from the Science and Engineering Research Board (SERB), Department of Science and Technology, Government of India. AD, AK, SNM and GS would like to acknowledge support from the project 5604-2 of the Indo-French Centre for the
Promotion of Advanced Research (IFCPAR). This work was supported by a research grant from the Center for Scientific Excellence at the Weizmann Institute of Science. AD, AK, SNM and GS acknowledge the hospitality of the Weizmann Institute during the SRITP Workshop "Correlations, Fluctuations and Anomalous Transport in Systems Far from Thermal Equilibrium" held at the Weizmann Institute in January 2018. SNM acknowledges the hospitality of the Weizmann Institute during a visit as a Weston Professor in 2019 and the support from the Science and Engineering Research Board (SERB, government of India), under the VAJRA faculty scheme (Ref. VJR/2017/000110) during a visit to Raman Research Institute, where part of this work was carried out. We would like to thank the ICTS program "Universality in random structures: Interfaces, Matrices, Sandpiles (Code: ICTS/URS2019/01)" for enabling valuable discussions with many participants. AK acknowledges support from DST grant under project No. ECR/2017/000634.
1 M. Riesz, Acta Sci. Math. Univ. Szeged, 9, 1 (1948).

2 N. S. Landkof, Foundations of modern potential theory (Vol. 180), Springer (1972).

3 D. Chafaï, N. Gozlan, P. A. Zitt, Ann. App. Probab. 24, 2371 (2014).

4 T. Leblé, S. Serfaty, Invent. Math. 210, 645 (2017).

5 P. J. Forrester, Log-gases and random matrices (Princeton University Press, Princeton, NJ 2010).

6 A. Lenard, J. Math. Phys. 2, 682 (1961).

7 S. Prager, Adv. Chem. Phys. 4, 201 (1962).

${ }^{8}$ R. J. Baxter, Proc. Camb. Phil. Soc. 59, 779 (1963).

9 A. Dhar, A. Kundu, S. N. Majumdar, S. Sabhapandit, G. Schehr, Phys. Rev. Lett. 119, 060601 (2017).

10 A. Dhar, A. Kundu, S. N. Majumdar, S. Sabhapandit, G. Schehr, J. Phys. A: Math. Theor. 51, 295001 (2018).

11 F. D. Cunden, P. Facchi, M. Ligabò, P. Vivo, J. Stat. Mech. P053303 (2017).

12 F. J. Dyson, J. Math. Phys. 3, 140; ibid 157; ibid 166 (1962).

13 M. L. Mehta, Random Matrices (Academic Press, Amsterdam, 2004).

14 The Oxford Handbook of Random Matrix Theory ed. by G. Akemann, G. Baik, and P. Di Francesco (Oxford University Press, Oxford, UK, 2011).

15 G. Livan, M. Novaes, and P. Vivo, Introduction to Random Matrices - Theory and Practice (Springer, New York, 2018).

16 I. Dumitriu, A. Edleman, J. Math. Phys. 43, 5830 (2002).

17 E. P. Wigner, Proc. Cambridge Philos. Soc. 47, 790 (1951).

18 M. Prähofer, H. Spohn, Phys. Rev. Lett. 84, 4882 (2000); M. Prähofer, H. Spohn, J. Stat. Phys. 108, 1071 (2002).

19 R. Marino, S. N. Majumdar, G. Schehr, P. Vivo, Phys. Rev. Lett. 112, 254101 (2014)

20 D. S. Dean, P. Le Doussal, S. N. Majumdar, G. Schehr, Phys. Rev. A 94, 063622 (2016).

21 D. S. Dean, P. Le Doussal, S. N. Majumdar, G. Schehr, J. Phys. A: Math. Theor. 52, 144006 (2019).
22 Y. Castin, arXiv:0612613, in Ultra-cold Fermi Gases, ed. by M. Inguscio, W. Ketterle, C. Salomon, (2006).

23 M. E. Fisher, J. Stat. Phys. 34, 667 (1984).

24 G. Schehr, S. N. Majumdar, A. Comtet, J. RandonFurling, Phys. Rev. Lett. 101, 150601 (2008).

25 J. Bun, J.-P. Bouchaud, S. N. Majumdar, M. Potters, Phys. Rev. Lett. 113, 070201 (2014).

${ }^{26}$ F. Calogero, J. Math. Phy. 10, 2197 (1969).

27 F. Calogero, J. Math. Phys. 12, 419 (1971).

28 F. Calogero, Lett. Nuovo Cimento 13, 411 (1975).

29 J. Moser, in Surveys in Applied Mathematics (Elsevier, 1976), pp. 235-258.

30 A. P. Polychronakos, J. Phys. A: Math. Gen. 39, 12793 (2006).

31 F. Calogero, J. Math. Phys. 22, 919 (1981).

32 S. Agarwal, M. Kulkarni, A. Dhar, preprint arXiv: 1903.09380

33 M. Lu, N. Q. Burdick, S. H. Youn, B. L. Lev, Phys. Rev. Lett. 107, 190401 (2011).

34 A. Griesmaier, J. Werner, S. Hensler, J. Stuhler, T. Pfau, Phys. Rev. Lett. 94, 160401 (2005).

35 K.-K. Ni, S. Ospelkaus, D. Wang, G. Quéméner, B. Neyenhuis, M. De Miranda, J. Bohn, J. Ye, D. Jin, Nature 464, 1324 (2010).

36 F. D. Cunden, P. Facchi, M. Ligabò, P. Vivo, J. Phys. A: Math. Theor. 51, 35LT01 (2018).

37 J. M. Brown A. Carrington, Rotational spectroscopy of diatomic molecules (Cambridge University Press, 2003).

38 These results are valid at sufficiently low temperature, i.e. for $\beta \gg N^{-2 \alpha_{k}}$, where $\alpha_{k}$ is given in Eq. (4). For $\beta=O\left(N^{-2 \alpha_{k}}\right)$, it is no longer sufficient to minimise just the energy and one has to take into account the contributions from the entropy, which will modify the average density. The relative importance of the energy and the entropy terms has been discussed in several previous works, see e.g. ${ }^{39-41}$. The entropy modified density was computed explicitly for the log-gas, i.e. in the $k \rightarrow 0^{+}$limit $^{42}$, and 
also for the eigenvalue density of the Wishart-Laguerre matrices $^{43}$. For another interesting recent application in the context of generalized hydrodynamics see Ref. ${ }^{44}$.

39 M. K. H. Kiessling, H. Spohn, Commun. Math. Phys. 199, 683 (1999).

40 F. D. Cunden, P. Facchi, P. Vivo, J. Phys. A 49(13), 135202 (2016).

${ }^{41}$ F. D. Cunden, P. Facchi, M. Ligabò, P. Vivo, J. Stat. Phys. 175, 1262 (2019)

42 R. Allez, J.-P. Bouchaud, A. Guionnet, Phys. Rev. Lett. 109, 094102 (2012).

43 R. Allez, J. P. Bouchaud, S. N. Majumdar, P. Vivo, J. Phys. A 46, 015001 (2012).

44 H. Spohn, preprint arXiv:1902.07751.

45 See Supplemental Material, which includes Refs. ${ }^{46,47}$, for more details on the computations.

${ }^{46}$ F. G. Tricomi, Integral equations, Dover publications (1985).

47 S. N. Majumdar, G. Schehr, J. Stat. Mech. P01012 (2014).

48 D. S. Dean and S. N. Majumdar, Phys. Rev. Lett. 97, 160201 (2006).

49 D. S. Dean and S. N. Majumdar, Phys. Rev. E 77, 041108 (2008).

50 E. B. Saff, V. Totik, Logarithmic potentials with exter- nal fields, (Vol. 316), Springer Science \& Business Media (2013).

51 S. Serfaty, Coulomb gases and Ginzburg-Landau vortices, Zurich Lectures in Advanced Mathematics, European Mathematical Society (EMS), Zürich (2015).

52 N. Ya Sonin, Studies of Cylinder functions and special polynomials (Gostekhizdat, Moscow, 1954) (in Russian).

53 G. Y. Popov, The elastic stress' concentration around dies, cuts, thin inclusions and reinforcements (1982).

54 H. Widom, J. Stat. Phys. 94, 347 (1999).

55 S. V. Buldyrev, M. Gitterman, S. Havlin, A. Ya. Kazakov, M. G. E. da Luz, E. P. Raposo, H. E. Stanley, G. M. Viswanathan, Physica A, 302, 148 (2001); PRE, 64, 041108 (2001).

56 B. Derrida, J. Stat. Mech. P07023 (2017).

57 J. Cividini, A. Kundu, A. Miron, D. Mukamel, J. Stat. Mech. P013203 (2017).

58 A. Miron, arXiv: 1902.08974

59 C. A. Tracy and H. Widom, Commun. Math. Phys. 159, 151 (1994); C. A. Tracy and H. Widom, Commun. Math. Phys. 177, 727 (1996).

60 D. P. Hardin, T. Leblé, E. B. Saff, S. Serfaty, Constr. Approx., 48, 61 (2018). 


\title{
Supplementary Material to "Harmonically confined particles with long-range repulsive interactions"
}

\author{
S. Agarwal,,${ }^{1,2}$ A. Dhar, ${ }^{1}$ M. Kulkarni, ${ }^{1}$ A. Kundu, ${ }^{1}$ S. N. Majumdar,${ }^{3}$ D. Mukamel,${ }^{4}$ and G. Schehr ${ }^{3}$ \\ ${ }^{1}$ International Centre for Theoretical Sciences, Tata Institute of Fundamental Research, Bengaluru - 560089, India \\ ${ }^{2}$ Birla Institute of Technology and Science, Pilani - 333031, India \\ ${ }^{3}$ LPTMS, CNRS, Univ. Paris-Sud, Universite Paris-Saclay, 91405 Orsay, France \\ ${ }^{4}$ Department of Physics of Complex Systems, Weizmann Institute of Science, Rehovot 7610001, Israel
}

(Dated: July 23, 2019)

\section{CONTINUUM APPROXIMATION FOR THE INTERACTION TERM}

In Eq. (1) of the main text, we defined the energy of a microscopic configuration $\left\{x_{i}\right\}$ as

$$
E\left[\left\{x_{i}\right\}\right]=\frac{1}{2} \sum_{i=1}^{N} x_{i}^{2}+\frac{J \operatorname{sgn}(k)}{2} \sum_{i \neq j} \frac{1}{\left|x_{i}-x_{j}\right|^{k}},
$$

where we assume $k>-2$. The partition function is defined as $Z_{N}(\beta)=\int \prod_{i=1}^{N} d x_{i} e^{-\beta E\left[\left\{x_{i}\right\}\right]}$. It turns out to be convenient to order the particle positions $x_{i}$ 's such that $x_{i}$ increases monotonically with the label $i$. Since the energy $E\left[\left\{x_{i}\right\}\right]$ in (1) is invariant under any permuation of the labels, it follows simply that

$$
Z_{N}(\beta)=N ! \iint_{x_{1}<x_{2}<\ldots<x_{N}} \ldots \int_{1} d x_{1} d x_{2} \ldots d x_{N} e^{-\beta E\left[\left\{x_{i}\right\}\right]} .
$$

Evaluating this microscopic $N$-fold integral in (2) exactly for arbitrary $N$ is hard. However, one can make progress in the large $N$ limit where one can coarse grain the system in terms of a smooth macroscopic density profile $\rho_{N}(x)$. Then the idea is to perform the integral in (2) in two steps. First, one fixes the macroscopic non-negative density $\rho_{N}(x)$ (normalized to unity), and integrates over all microscopic configurations in the partition sum (2) compatible with this macroscopic density $\rho_{N}(x)$. Secondly, one integrates over all possible smooth macroscopic density profiles $\rho_{N}(x)$-leading to a functional integral over $\rho_{N}(x)$.

It turns out (as we show below) that the energy in (1) can be expressed as a functional over only this macroscopic density, i.e., $E\left[\left\{x_{i}\right\}\right] \approx \mathcal{E}\left[\rho_{N}(x)\right]$, to leading order for large $N$. Then, the partition function in $(2)$ can be approximated as

$$
Z_{N}(\beta) \approx N ! \int \mathcal{D}\left[\rho_{N}(x)\right] \mathcal{N}\left[\rho_{N}(x)\right] e^{-\beta \mathcal{E}\left[\rho_{N}(x)\right]} \delta\left(\int d x \rho_{N}(x)-1\right)
$$

where the delta function enforces the normalization of the macroscopic density (to unity) and $\mathcal{N}\left[\rho_{N}(x)\right]$ is a combinatorial factor that counts the number of microscopic configurations compatible with a given macroscopic profile $\rho_{N}(x)$. Hence $\mathcal{N}\left[\rho_{N}(x)\right]=\exp \left[S\left[\rho_{N}(x)\right]\right]$, where $S\left[\rho_{N}(x)\right]$ is the entropy associated with the macroscopic configuration $\rho_{N}(x)$. One can explicitly compute the entropy term and show that $S\left[\rho_{N}(x)\right]=-N \int d x \rho_{N}(x) \ln \left[\rho_{N}(x)\right]$ up to an additive constant (for a simple derivation in the context of the log-gas, see e.g. Refs. 1,2). Furthermore, the delta function enforcing the normalization constraint can be replaced by its integral representation, $\delta(y)=\int \frac{d \mu}{2 \pi} e^{-\mu y}$ where the integral is along the imaginary $\mu$ axis. Putting all these together on the right hand side (rhs), we obtain, up to an overall (unimportant) multiplicative factor,

$$
Z_{N}(\beta) \sim \int d \mu \int \mathcal{D}\left[\rho_{N}(x)\right] \exp \left[-\beta \mathcal{E}\left[\rho_{N}(x)\right]-\mu\left(\int \rho_{N}(x) d x-1\right)-N \int d x \rho_{N}(x) \ln \left[\rho_{N}(x)\right]\right] .
$$

It will turn out that for large $N$ and fixed $k>-2$, the energy term will scale as $N^{b_{k}}$ with $b_{k}>1$, compared to the entropy term $(\sim O(N))$. Hence the energy dominates for all $k>-2$ and one can ignore the entropy term for large $N$. Ignoring the entropy term leads to

$$
Z_{N}(\beta) \sim \int d \mu \int \mathcal{D}\left[\rho_{N}(x)\right] \exp \left[-\beta \mathcal{E}\left[\rho_{N}(x)\right]-\mu\left(\int \rho_{N}(x) d x-1\right)\right] .
$$


Thus, our next task is to show that indeed the energy $E\left[\left\{x_{i}\right\}\right]$ can be approximated by $\mathcal{E}\left[\rho_{N}(x)\right]$, a functional of only the macroscopic density $\rho_{N}(x)$. This is far from obvious, in particular for the interaction term (the second term) on the rhs of (1). We now show how it can be done. It turns out that the form of $\mathcal{E}\left[\rho_{N}(x)\right]$ is very different for the three cases: (i) $-2<k<1$ (ii) $k>1$ and (iii) $k=1$. To proceed, the first step is to approximate the microscopic ordered coordinate $x_{i}$ by a smooth monotonically increasing function $x(s)$ in the large $N$ limit, such that $x(i)=x_{i}$. One can relate this smooth function $x(s)$ to the local macroscopic density by noting that

$$
N \int^{x} \rho_{N}\left(x^{\prime}\right) d x^{\prime}=s(x),
$$

which follows from the fact that the total number of particles up to label $s$ is simply $s$. Taking a further derivative of (6) with respect to $x$ yields

$$
\frac{d x}{d s}=\frac{1}{N \rho_{N}(x(s))} .
$$

Note that this relation (7) is true only for ordered configurations such that $d x / d s>0$. This is needed since the rhs of (7) is positive because the density is manifestly positive. The average of any function $f\left(x_{i}\right)$ of the coordinates $x_{i}$, can then be expressed in terms of the macroscopic density as

$$
\sum_{i=1}^{N} f\left(x_{i}\right) \approx \int f(x) d s=N \int f(x) \rho_{N}(x) d x
$$

which holds for any smooth function $f(x)$. For example, using $f(x)=x^{2}$ in (8), the first term (corresponding to the external harmonic potential) on the rhs of (1) can be simply expressed as a functional of $\rho_{N}(x)$

$$
\mathcal{E}_{\text {harmonic }}\left[\rho_{N}(x)\right] \approx \frac{N}{2} \int x^{2} \rho_{N}(x) d x
$$

where the integral runs over the support of $\rho_{N}(x)$, i.e., where $\rho_{N}(x)$ is nonzero.

The next step is to express the second term (interaction term) as a functional of $\rho_{N}(x)$. This requires a bit more work. We proceed by replacing $x_{i}-x_{j}=x(i)-x(j)$ in the interaction term in (1) and express it first as a functional of $x(s)$ as

$$
\mathcal{E}_{\mathrm{int}}[\{x(s)\}] \approx \frac{J \operatorname{sgn}(k)}{2} \sum_{\substack{i, j=1 \\ i \neq j}}^{N} \frac{1}{|x(i)-x(j)|^{k}} .
$$

The challenge now is to express this energy in (10) as a functional of $\rho_{N}(x)$, instead of a functional of $x(s)$. We now consider the two cases $-2<k<1$ and $k>1$ separately. This task turns out to be simpler in the former case than the latter one. Later, we treat the marginal case $k=1$ separately.

\section{A. The case $-2<k<1$}

In this case, the double sum in (10) can be replaced, to leading order for large $N$, by a double integral using the identity (8) twice and the resulting integral is convergent. This leads to

$$
\mathcal{E}_{\mathrm{int}}\left[\rho_{N}(x)\right] \approx \frac{J \operatorname{sgn}(k)}{2} N^{2} \iint \frac{\rho_{N}(x) \rho_{N}\left(x^{\prime}\right)}{\left|x-x^{\prime}\right|^{k}} d x d x^{\prime}
$$

The correction terms are subleading to this leading $O\left(N^{2}\right)$ term. Adding (9) and (11), we obtain the desired coarse grained energy as a functional of the macroscopic density $\rho_{N}(x)$

$$
\mathcal{E}\left[\rho_{N}(x)\right] \approx \frac{N}{2} \int x^{2} \rho_{N}(x) d x+\frac{J \operatorname{sgn}(k)}{2} N^{2} \iint \frac{\rho_{N}(x) \rho_{N}\left(x^{\prime}\right)}{\left|x-x^{\prime}\right|^{k}} d x d x^{\prime} .
$$

We then rescale $x=N^{\alpha_{k}} y$ and write $\rho_{N}(x)=N^{-\alpha_{k}} \tilde{\rho}_{k}\left(x N^{-\alpha_{k}}\right)$, where $\int \tilde{\rho}_{k}(y) d y=1$ due to the normalization. Demanding that both terms scale as the same power of $N$ fixes $\alpha_{k}=1 /(k+2)$ as stated in the first line of Eq. (4) 
in the main text. Consequently, the total energy scales as $N^{b_{k}}$, where $b_{k}=(4+k) /(2+k)$. Note that $b_{k}>1$ for $k>-2$. Consequently, dropping the entropy term in (5) is justified a posteriori. Furthermore, rescaling $\mu \rightarrow \mu N^{b_{k}}$, we can write the partition function in (5) as

$$
Z_{N}(\beta) \sim \int d \mu \int \mathcal{D}\left[\tilde{\rho}_{k}\right] \exp \left(-\beta N^{\frac{4+k}{2+k}} \Sigma\left[\tilde{\rho}_{k}(y)\right]\right)
$$

where the action $\Sigma\left[\tilde{\rho}_{k}(y)\right]$ is given by

$$
\Sigma\left[\tilde{\rho}_{k}(y)\right]=\frac{J \operatorname{sgn}(k)}{2} \int d y \int d y^{\prime} \frac{\tilde{\rho}_{k}(y) \tilde{\rho}_{k}\left(y^{\prime}\right)}{\left|y-y^{\prime}\right|^{k}}+\frac{1}{2} \int d y y^{2} \tilde{\rho}_{k}(y)-\mu\left(\int d y \tilde{\rho}_{k}(y)-1\right) .
$$

This completes the derivation of Eqs. (7) and (8) in the main text. Taking functional derivative $\frac{\left.\partial \Sigma\left[\tilde{\rho}_{k}(y)\right]\right]}{\partial \tilde{\rho}_{k}(y)}$ of the action in (14) and setting it to zero gives Eq. (9) of the main text, i.e.,

$$
\frac{y^{2}}{2}+J \operatorname{sgn}(k) \int d y^{\prime} \frac{\tilde{\rho}_{k}\left(y^{\prime}\right)}{\left|y-y^{\prime}\right|^{k}}=\mu .
$$

Finally, taking one more derivative with respect to $y$ inside the support of $\tilde{\rho}_{k}(y)$ gives the singular integral equation in (10) of the main text. The solution of this integral equation is detailed later in Section II of this Supplementary Material.

\section{B. The case $k>1$}

This case turns out to be harder due to the strong singularity of the rhs of (10) in the vicinity of $i=j$. Consequently, we can no longer replace the double sum by the double integral using the identity $(8)$ as we did for $-2<k<1$. The double integral is simply divergent. Thus in this case, the double sum needs to be evaluated more carefully by separating out the singular (nonintegrable) and the regular (integrable) parts of $|x(i)-x(j)|^{-k}$ as $j \rightarrow i$. To proceed, we first expand $x(j)$ in a Taylor series for $j$ close to $i$

$$
x(j)-x(i)=\sum_{n=1}^{\infty} \frac{(j-i)^{n}}{n !} x^{[n]}(i)
$$

where $x^{[n]}(i)=\left.\frac{d^{n} x(s)}{d s^{n}}\right|_{s=i}$ is the $n$-th derivative of the smooth function $x(s)$ at $s=i$. Using (16), one can then formally express $|x(i)-x(j)|^{-k}$ also as a series

$$
\frac{1}{|x(i)-x(j)|^{k}}=\sum_{m=0}^{\infty} \frac{a_{m, k}}{|j-i|^{k-m}}
$$

where the coefficients $a_{m, k}$ 's can be easily computed in terms of the derivatives $x^{[n]}(i)$. We note that in this expansion the terms up to $m=m^{*}=\operatorname{Int}(k-1)$ (i.e., the integer part of $\left.(k-1)\right)$ give rise to non-integrable singularities, while the rest of the terms are regular, i.e., integrable. Hence we can separate these two contributions and write

$$
\frac{1}{|x(i)-x(j)|^{k}}=\sum_{m=0}^{m^{*}} \frac{a_{m, k}}{|j-i|^{k-m}}+R(x(i), x(j))
$$

where $R(x(i), x(j))$ denotes the integrable 'remainder' part. We then substitute this expansion (18) on the rhs of (10) to express the interaction energy as

$$
\mathcal{E}_{\mathrm{int}}[\{x(s)\}] \approx \frac{J}{2} \sum_{\substack{i, j=1 \\ i \neq j}}^{N}\left[\sum_{m=0}^{m^{*}} \frac{a_{m, k}}{|j-i|^{k-m}}+R(x(i), x(j))\right] .
$$

For large $N$, the double sum over the regular integrable part can again be replaced by a double integral using the identity (8) twice

$$
\frac{J}{2} \sum_{\substack{i, j=1 \\ i \neq j}}^{N} R(x(i), x(j)) \approx \frac{J}{2} N^{2} \iint d x d x^{\prime} R\left(x, x^{\prime}\right) \rho_{N}(x) \rho_{N}\left(x^{\prime}\right)
$$


and hence it scales as $N^{2}$ for large $N$.

The singular terms on the right hand side of (19) have to be evaluated separately. Consider first the term corresponding to $m=0$. It is easy to see that

$$
a_{0, k}=\left[x^{\prime}(i)\right]^{-k}=\left[N \rho_{N}(x(i))\right]^{k},
$$

where we used (7). Then the first singular term on the right hand side of (19) reads

$$
T_{1}(\text { singular })=\frac{J}{2} \sum_{\substack{i, j=1 \\ i \neq j}}^{N} \frac{\left[N \rho_{N}(x(i))\right]^{k}}{|j-i|^{k}} .
$$

Next we perform the sum over $j$ keeping $i$ fixed. For large $N$, to leading order, this sum over $n=j-i$ can be extended from $-\infty$ to $\infty$, excluding $n=j-i=0$. This gives, using the symmetry factor 2 ,

$$
T_{1}(\text { singular })=\sum_{j \neq i} \frac{1}{|j-i|^{k}} \approx 2 \sum_{k=1}^{\infty} \frac{1}{n^{k}}=2 \zeta(k),
$$

where the Riemann zeta function $\zeta(k)$ is finite for $k>1$. Furthermore, the sum over $i$ in (22) can now be replaced by an integral using again the identity (8). This gives finally

$$
\frac{J}{2} \sum_{\substack{i, j=1 \\ i \neq j}}^{N} \frac{\left[N \rho_{N}(x(i))\right]^{k}}{|j-i|^{k}} \approx J \zeta(k) N^{k+1} \int\left[\rho_{N}(x)\right]^{k+1} d x .
$$

Note that this term scales as $N^{k+1}$ for large $N$ which is larger than $O\left(N^{2}\right)$ in $(20)$ for $k>1$. Hence the singular term $T_{1}$ corresponding to $m=0$ dominates over the regular term in (19) for large $N$. One can similarly evaluate the next singular terms $m=1,2, \ldots, m^{*}$. For instance, for $m=1$, one gets, from Eq. (17)

$$
a_{1, k}=-\frac{k}{2} \frac{x^{\prime \prime}(i)}{\left[x^{\prime}(i)\right]^{k+1}} .
$$

Differentiating Eq. (7) with respect to $s$ and using $x^{\prime}(s)=\left[N \rho_{N}(x(s))\right]^{-1}$ one gets

$$
x^{\prime \prime}(s)=-\frac{\rho_{N}^{\prime}(s)}{N^{2}\left[\rho_{N}(s)\right]^{3}} .
$$

Hence $a_{1, k}$ in (25) reads

$$
a_{1, k}=\frac{k}{2} N^{k-1}\left[\rho_{N}(x(i))\right]^{k-2} \rho_{N}^{\prime}(x(i)) .
$$

Hence the contribution for the $m=1$ term is given by

$$
T_{2} \text { (singular) }=\frac{J k}{4} N^{k-1} \sum_{i \neq j} \frac{\left[\rho_{N}(x(i))\right]^{k-2} \rho_{N}^{\prime}(x(i))}{|i-j|^{k-1}} .
$$

Performing the double sum as in the $m=0$ case, it is easy to see that $T_{2}$ (singular) $\sim N^{k}$ for large $N$. For $k>1$, this is clearly smaller the singular term corresponding to $m=0$ which is of order $O\left(N^{k+1}\right)$. One can similarly evaluate the next order terms and show that, for $k>1$, they are all subdominant, compared to the leading $m=0$ term. Hence, finally, we find that to leading order for large $N$ and $k>1$

$$
\mathcal{E}_{\mathrm{int}}[\{x(s)\}] \approx J \zeta(k) N^{k+1} \int\left[\rho_{N}(x)\right]^{k+1} d x .
$$

Adding this to the harmonic part in (9) then gives the leading large $N$ behavior of the total energy for $k>1$

$$
\mathcal{E}\left[\rho_{N}(x)\right] \approx \frac{N}{2} \int x^{2} \rho_{N}(x) d x+J \zeta(k) N^{k+1} \int\left[\rho_{N}(x)\right]^{k+1} d x,
$$


as stated in Eq. (11) of the main text. Comparing (12) for $-2<k<1$ and (30) for $k>1$, we see that while in the former case the leading large $N$ behavior of the total energy is a non-local functional of the density $\rho_{N}(x)$, in the latter case it is completely local.

As in the case $-2<k<1$, we next rescale $x=N^{\alpha_{k}} y$ in (30) and write $\rho_{N}(x)=N^{-\alpha_{k}} \tilde{\rho}_{k}\left(x N^{-\alpha_{k}}\right)$, where $\int \tilde{\rho}_{k}(y) d y=1$ due to the normalization. We demand that both terms on the rhs of (30) scale as the same power of $N$ for large $N$. This fixes the exponent $\alpha_{k}=k /(k+2)$, as stated in the second line of Eq. (4) in the main text. Hence, the total energy scales as $N^{b_{k}}$ where $b_{k}=(3 k+2) /(k+2)$. Once again, $b_{k}>1$ for $k>1$, justifying the fact that we have dropped the entropy term $(\sim O(N))$ in (5). Again, rescaling $\mu \rightarrow \mu N^{b_{k}}$ in (5) we get, to leading order for large $N$

$$
Z_{N}(\beta) \sim \int d \mu \int \mathcal{D}\left[\tilde{\rho}_{k}\right] \exp \left(-\beta N^{\frac{3 k+2}{k+2}} \Sigma\left[\tilde{\rho}_{k}(y)\right]\right)
$$

where the action $\Sigma\left[\tilde{\rho}_{k}(y)\right]$ is given by

$$
\Sigma\left[\tilde{\rho}_{k}(y)\right]=\frac{1}{2} \int d y y^{2} \tilde{\rho}_{k}(y)+J \zeta(k) \int d y\left[\tilde{\rho}_{k}(y)\right]^{k+1}-\mu\left(\int d y \tilde{\rho}_{k}(y)-1\right) .
$$

This then completes the derivation of Eqs. (12) and (13) in the main text.

Minimizing the action in (32) gives the saddle point equation that reads

$$
\frac{y^{2}}{2}+J \zeta(k)(k+1)\left[\tilde{\rho}_{k}(y)\right]^{k}=\mu
$$

where the Lagrange multiplier $\mu$ enforces the normalization $\int \tilde{\rho}_{k}(y) d y=1$. Solving Eq. (33) trivially gives

$$
\tilde{\rho}_{k}(y)=[2 J \zeta(k)(k+1)]^{-1 / k}\left(2 \mu-y^{2}\right)^{1 / k} ; \quad-\sqrt{2 \mu} \leq y \leq \sqrt{2 \mu} .
$$

The constant $\mu$ is fixed from the normalization condition

$$
\int_{-\sqrt{2 \mu}}^{\sqrt{2 \mu}} \tilde{\rho}_{k}(y) d y=1
$$

Performing this integral and setting the support length $\ell_{k}=2 \sqrt{2 \mu}$ gives

$$
\ell_{k}=\left[\frac{(2 J \zeta(k)(k+1))^{1 / k}}{B(1+1 / k, 1+1 / k)}\right]^{\frac{k}{k+2}}
$$

where $B(a, b)$ is the standard Beta function.

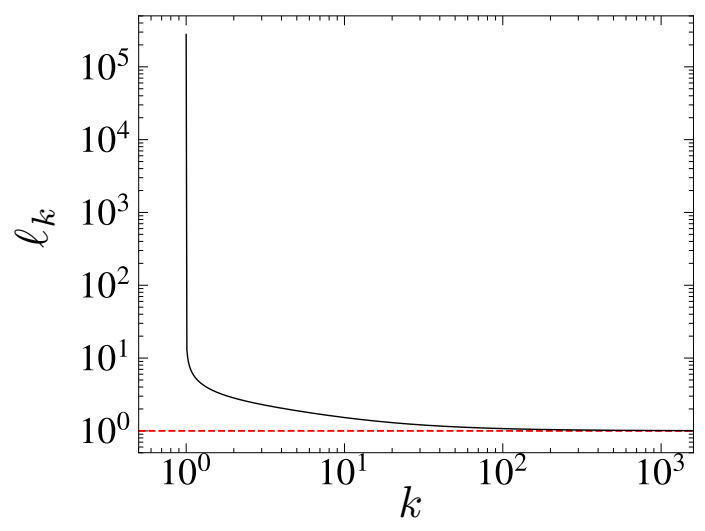

FIG. 1: The support length $\ell_{k}$ in (36) is plotted here as a function of $k$, for the case $k>1$. As $k$ increases beyond 1 , the support length $\ell_{k}$ decreases monotonically and approaches 1 as $k \rightarrow \infty$.

Finally, the solution $\tilde{\rho}_{k}(y)$ can be expressed as $\tilde{\rho}_{k}(y)=\left(1 / \ell_{k}\right) F_{k}\left(y / \ell_{k}\right)$ where the scaling function $F_{k}(z)$ is supported over $z \in[-1 / 2,1 / 2]$ and is given by

$$
F_{k}(z)=\frac{1}{B\left(\gamma_{k}+1, \gamma_{k}+1\right)}\left(\frac{1}{4}-z^{2}\right)^{\gamma_{k}}
$$

as stated in Eq. (5) of the main text with the exponent $\gamma_{k}=1 / k$, as given in the second line of Eq. (6) of the main text. 


\section{The marginal case $k=1$}

For the marginal case $k=1$, let us first evaluate the singular part of the interaction energy in (10). The Taylor expansion of $x(j)$ around $j=i$ as in (18) yields

$$
\frac{1}{|x(i)-x(j)|}=\frac{1}{|j-i|\left|x^{\prime}(i)\right|}+R(x(i), x(j)),
$$

where $R(x(i), x(j))$ is again the regular integrable part. Substituting this in (10), the double sum over the integrable part can again be replaced by the double integral as in (20) and this term scales as $\sim N^{2}$ for large $N$. The contribution from the leading singular term reads

$$
T_{1} \text { (singular) }=\frac{J}{2} \sum_{\substack{i, j=1 \\ i \neq j}}^{N} \frac{\left[N \rho_{N}(x(i))\right]}{|j-i|},
$$

where we used the relation $x^{\prime}(i)=1 /\left[N \rho_{N}(x(i))\right]$. But now the sum over $j$, for fixed $i$, is not convergent. For large $N$, this sum over $j$ can be extended from $i-N / 2$ to $i+N / 2$ and hence to leading order for large $N$ it can be approximated by $\sum_{j \neq i} 1 /|j-i| \approx 2 \ln N$. Replacing further the single sum over $i$ by an integral using the identity (8) gives the large $N$ estimate

$$
T_{1}(\text { singular })=\frac{J}{2} \sum_{\substack{i, j=1 \\ i \neq j}}^{N} \frac{\left[N \rho_{N}(x(i))\right]}{|j-i|} \approx J N^{2} \ln N \int\left[\rho_{N}(x)\right]^{2} d x .
$$

This term thus scales as $\sim N^{2} \ln N$ and hence dominates over the $O\left(N^{2}\right)$ behavior of the integrable remainder part. Hence, to leading order for large $N$, we get the interaction energy

$$
\mathcal{E}_{\mathrm{int}}[\{x(s)\}] \approx J N^{2} \ln N \int\left[\rho_{N}(x)\right]^{2} d x .
$$

Adding this to the harmonic part in (9) then gives the leading large $N$ behavior of the total energy for $k=1$

$$
\mathcal{E}\left[\rho_{N}(x)\right] \approx \frac{N}{2} \int x^{2} \rho_{N}(x) d x+J N^{2} \ln N \int\left[\rho_{N}(x)\right]^{2} d x .
$$

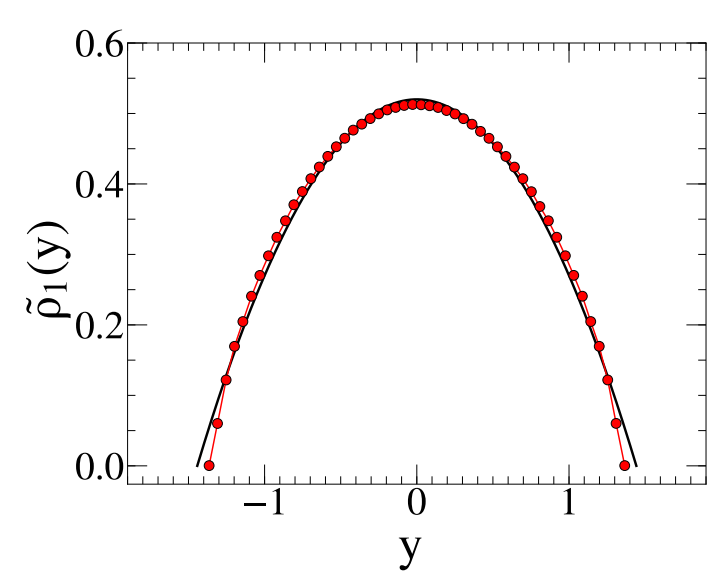

FIG. 2: The numerical (Monte Carlo) average scaled density $\tilde{\rho}_{1}(y)$ vs $y$ for $k=1$, with parameters $N=200, J=1$ and $\beta=2$ (red circles) compared to the prediction in (47) (black line). The agreement is excellent. The Monte Carlo average is over $2 \times 10^{8}$ samples.

Next, we rescale $x=L_{N} y$ and write $\rho_{N}(x)=\left(1 / L_{N}\right) \tilde{\rho}_{1}\left(x / L_{N}\right)$, where $L_{N}$ is yet to be determined. Substituting this scaling form in (42), we find that the first term scales as $N L_{N}^{2}$, while the interaction term scales as $N^{2} \ln N / L_{N}$. Demanding that they are of the same order indicates that to leading order for large $N$

$$
L_{N} \approx(N \ln N)^{1 / 3} .
$$


The total energy then scales as $E \sim N^{5 / 3}(\ln N)^{2 / 3}$, which again is larger than the entropy term $(\sim O(N))$, hence justfying the fact that we have dropped the entropy term in (5). Hence, the partition function $Z_{N}(\beta)$ in $(5)$ for $k=1$ can be expressed, to leading order for large $N$, as

$$
Z_{N}(\beta) \sim \int d \mu \int \mathcal{D}\left[\tilde{\rho}_{1}(y)\right] \exp \left(-\beta N^{5 / 3}(\ln N)^{2 / 3} \Sigma\left[\tilde{\rho}_{1}(y)\right]\right),
$$

where the coarse grained action $\Sigma\left[\tilde{\rho}_{1}(y)\right]$ is given by

$$
\Sigma\left[\tilde{\rho}_{1}(y)\right]=\frac{1}{2} \int d y y^{2} \tilde{\rho}_{1}(y)+J \int\left[\tilde{\rho}_{1}(y)\right]^{2} d y-\mu\left(\int d y \tilde{\rho}_{1}(y)-1\right) .
$$

Here again $\mu$ is the Lagrange multiplier that enforces the normalization constraint $\int d y \tilde{\rho}_{1}(y)=1$. The partition function can then be evaluated by the saddle point method for large $N$. Minimizing the action in (45) with respect to $\tilde{\rho}_{1}(y)$ yields the saddle point equation

$$
\frac{y^{2}}{2}+2 J \tilde{\rho}_{1}(y)=\mu
$$

Solving this equation trivially gives

$$
\tilde{\rho}_{1}(y)=\frac{1}{4 J}\left(2 \mu-y^{2}\right) ; \quad-\sqrt{2 \mu} \leq y \leq \sqrt{2 \mu} .
$$

Finally, enforcing the normalization condition $\int_{-\sqrt{2 \mu}}^{\sqrt{2 \mu}} \tilde{\rho}_{1}(y) d y=1$ fixes the Lagrange multiplier

$$
\mu=\frac{1}{2}(3 J)^{2 / 3}
$$

Hence, the total support length $\ell_{1}$ is given by

$$
\ell_{1}=2 \sqrt{2 \mu}=2(3 J)^{1 / 3} .
$$

Consequently, the scaled density can be expressed as, $\tilde{\rho}_{1}(y)=\left(1 / \ell_{1}\right) F_{1}\left(y / \ell_{1}\right)$ where the scaling function

$$
F_{1}(z)=6\left(\frac{1}{4}-z^{2}\right) ; \quad-1 / 2 \leq z \leq 1 / 2
$$

which yields the form given in Eq. (5) in the main text with exponent $\gamma_{1}=1$. We have compared the analytical prediction (47) for $k=1$ with Monte Carlo simulation result (see Fig. 2 above) and found very good agreement.

\section{SOLUTION OF THE SINGULAR INTEGRAL EQUATION FOR $-2<k<1$}

In this section we provide a detailed exact solution of the singular integral equation (10) in the main text for $-2<k<1$. This equation reads

$$
P V \int_{-\ell_{k} / 2}^{\ell_{k} / 2} \frac{\operatorname{sgn}\left(y^{\prime}-y\right)}{\left|y-y^{\prime}\right|^{k+1}} \tilde{\rho}_{k}\left(y^{\prime}\right) d y^{\prime}=-\frac{y}{J|k|}, \quad k \neq 0
$$

where $P V$ stands for the principal value. Note that this integral is interpreted in a principal value sense only for $0<k<1$, but for $k<0$ it is an ordinary integral (since it is integarble for $k<0$ ). The goal is to invert this integral equation to obtain the scaled density $\tilde{\rho}_{k}(y)$ which is supported over $y \in\left[-\ell_{k} / 2, \ell_{k} / 2\right]$ and satisfies the normalization condition

$$
\int_{-\ell_{k} / 2}^{\ell_{k} / 2} \tilde{\rho}_{k}(y) d y=1
$$

Consider first the limit $k \rightarrow 0^{+}$, where we set $J|k|=1$. In this case Eq. (51) reduces to a singular integral equation of the Cauchy type (with one compact support over $y \in\left[a_{1}, a_{2}\right]$ )

$$
P V \int_{a_{1}}^{a_{2}} \frac{\tilde{\rho}_{0}\left(y^{\prime}\right)}{y-y^{\prime}} d y^{\prime}=g(y)
$$


where the source function $g(y)=y, a_{1}=l_{0} / 2$ and $a_{2}=-l_{0} / 2$. For an arbitrary source function $g(y)$ and for a compact single support $\left[a_{1}, a_{2}\right]$, the Cauchy singular equation (53) can be explicitly inverted to obtain the scaled density $\tilde{\rho}_{0}(y)$ using a formula due to Tricomi ${ }^{3}$ that reads

$$
\tilde{\rho}_{0}(y)=\frac{1}{\pi \sqrt{\left(a_{2}-y\right)\left(y-a_{1}\right)}}\left[B_{0}-P V \int_{a_{1}}^{a_{2}} \frac{d t}{\pi} g(t) \frac{\sqrt{\left(a_{2}-t\right)\left(t-a_{1}\right)}}{y-t}\right]
$$

where $B_{0}=\int_{a_{1}}^{a_{2}} \tilde{\rho}_{0}(y) d y$ (for a discussion of the Tricomi solution in the log-gas case, see section 3 of the review in Ref. 4). In our case, the source function $g(y)=y$, the support is symmetric around 0 , i.e., $a_{1}=-\ell_{0} / 2$ and $a_{2}=\ell_{0} / 2$ and the the normalization condition $\int_{-l_{0} / 2}^{l_{0} / 2} \tilde{\rho}_{0}(y) d y=1$ indicates $B_{0}=1$. Evaluating the $P V$ integral on the right hand side of Eq. (54) then gives the semi-circular law

$$
\tilde{\rho}_{0}(y)=\frac{1}{\pi} \sqrt{2-y^{2}} ; \quad-\sqrt{2} \leq y \leq \sqrt{2}
$$

and we also have $\ell_{0}=2 \sqrt{2}$. This solution can then be written as $\tilde{\rho}_{0}(y)=\left(1 / \ell_{0}\right) F_{0}\left(y / \ell_{0}\right)$, where $F_{0}(z)=\frac{8}{\pi} \sqrt{1 / 4-z^{2}}$ with $z \in[-1 / 2,1 / 2]$, consistent with the result in Eq. (5) of the main text with the exponent $\gamma_{0}=1 / 2$. To compare with Eq. (5) of the main text for $\gamma_{0}=1 / 2$, we used $B(3 / 2,3 / 2)=\pi / 8$.

The other case where the solution can be easily obtained corresponds to $k=-1$ in (51). In this case, we take one more derivative of Eq. (51) with respect to $y$ and use the relation: $\frac{d}{d y}\left[\operatorname{sgn}\left(y-y^{\prime}\right)\right]=2 \delta\left(y-y^{\prime}\right)$. This immediately gives a flat density, $\tilde{\rho}_{-1}(y)=1 / 2$ with support over $[-1,1]$. Thus the total support length $\ell_{-1}=2$. Once again, this density can be trivially expressed in the scaling form, $\tilde{\rho}_{-1}(y)=\left(1 / \ell_{-1}\right) F_{-1}\left(y / \ell_{-1}\right)$, with the scaling function $F_{-1}(z)=1$ over $z \in[-1 / 2,1 / 2]$. This is again consistent with the stated result in Eq. (5) of the main text with the exponent $\gamma_{-1}=0$.

Can we go beyond these two solvable cases namely the $k \rightarrow 0^{+}$limit and $k=-1$, and invert the integral equation (51) for a general $-2<k<1$ ? Fortunately, the answer is yes and there is an inversion formula due to Sonin ${ }^{5}$ which is a generalization of the Tricomi formula, as we explain now. To make progress for general $k$, it is useful to first make a change of variables $z=y+\ell_{k} / 2, z^{\prime}=y^{\prime}+\ell_{k} / 2$ in Eq. (51) to bring it into the standard Sonin form. Let us write the density in the $z$ variable as $d_{k}(z)=\tilde{\rho}_{k}\left(z-\ell_{k} / 2\right)$. In this $z$ variable the density $d_{k}(z)$ has support over the positive region $\left[0, \ell_{k}\right]$. With this change of variable, the integral equation $(51)$ reads

$$
P V \int_{0}^{\ell_{k}} \frac{\operatorname{sgn}\left(z^{\prime}-z\right)}{\left|z-z^{\prime}\right|^{p}} d_{k}\left(z^{\prime}\right) d z^{\prime}=h(z)
$$

where

$$
p=k+1, \quad \text { and } \quad h(z)=-\frac{1}{J|k|}\left(z-\ell_{k} / 2\right)
$$

It is in this form that this integral equation was first studied by Sonin ${ }^{5}$ for an arbitrary source function $h(z)$. Since then, this equation has appeared in various problems in the mathematics literature ${ }^{6,7}$ and also in a number of physical problems. For example, it appears in the computation of the mean exit time of a Lévy walker (of Lévy index $p$ ) from a finite interval $\left[0, \ell_{k}\right]$ in one dimension ${ }^{8,9}$. The other physical example concerns the computation of the temperature profile for systems with anomalous heat conduction in one dimension ${ }^{10,11}$. It turns out that it is indeed possible to invert this integral equation (56) and obtain $d_{k}(z)$ explicitly for arbitrary $h(z)$ and $-1<p<2$ (i.e., $-2<k<1$ ) this is known as the Sonin inversion formula ${ }^{5}$. The general solution of (56) can be expressed as a linear combination ${ }^{8}$

$$
d_{k}(z)=c_{0}\left[z\left(\ell_{k}-z\right)\right]^{p / 2-1}+u_{k}(z)
$$

where the first term is a homogeneous solution of $(56)$ with $h(z)=0, c_{0}$ is an arbitrary constant and $u_{k}(z)$ is a particular solution of (56) given explicitly by

$$
u_{k}(z)=\frac{2 \sin (\pi p / 2)}{\pi p B(p / 2, p / 2)} z^{p / 2-1} \times \frac{d}{d z} \int_{z}^{\ell_{k}} d t t^{1-p}(t-z)^{p / 2} \frac{d}{d t} \int_{0}^{t} y^{p / 2}(t-y)^{p / 2-1} h(y) d y .
$$

We insert $h(y)=-\frac{1}{J|k|}\left(y-\ell_{k} / 2\right)$ in the second term on the right hand side of Eq. (59) and perform the double integrals. After a few steps of straightforward algebra we get the explicit solution

$$
u_{k}(z)=\frac{1}{J|k|} \frac{\sin \left(\frac{\pi p}{2}\right)}{\pi p}\left[z\left(\ell_{k}-z\right)\right]^{p / 2},-1<p=k+1<2 .
$$


The general solution is therefore

$$
d_{k}(z)=c_{0}\left[z\left(\ell_{k}-z\right)\right]^{p / 2-1}+\frac{1}{J|k|} \frac{\sin \left(\frac{\pi p}{2}\right)}{\pi p}\left[z\left(\ell_{k}-z\right)\right]^{p / 2} .
$$

The constant $c_{0}$ in (61) has to be fixed by physical considerations. Indeed, the homogeneous solution $c_{0}\left[z\left(\ell_{k}-z\right)\right]^{p / 2-1}$ of the integral equation (56) is obtained when $h(z)=0$, i.e., when there is no confining potential. However, when there is no confining potential, physically it is clear that since the charges repel each other, there can not be a confining physical density-the charges will just fly off to infinite distance from each other to minimize the energy. So, even if there exists a mathematical solution of the integral equation (56) for $h(z)=0$, it can not correspond to a physical solution. It is a spurious solution that must be discarded. This physical argument sets the constant $c_{0}=0 .{ }^{13}$

One can also show this more rigorously as follows. Consider the solution $d_{k}(z)$ in (61) that has two parameters $c_{0}$ and $\ell_{k}$ that are yet to be determined. We recall that $d_{k}(z)$ must be normalized to unity [see Eq. (52)]

$$
\int_{0}^{\ell_{k}} d_{k}(z) d z=1
$$

This gives one relation between the two parameters $c_{0}$ and $\ell_{k}$. We need one more condition.

Consider first the case $-1<p=k+1<0$. In this case, while the particular solution $u_{k}(z)$ in Eq. (60) is integrable over $z \in\left[0, \ell_{k}\right]$, the homogeneous part $c_{0}\left[z\left(\ell_{k}-z\right)\right]^{p / 2-1}$ is not integrable over $z \in\left[0, \ell_{k}\right]$ and hence, to satisfy Eq. (62), we must have $c_{0}=0$. Next we consider the complementary case: $0<p=k+1<2$. This case is a bit harder. Here, even the homogeneous solution is integrable, so we can not use the same argument. However, note first that $c_{0}$ can not be negative because near the edges $z \rightarrow 0$ and $z \rightarrow \ell_{k}$, the homogeneous solution in (61) dominates over the particular solution $u_{k}(z)$ as it has stronger singularities at the edges. Hence, if $c_{0}$ is negative that would imply that the density is negative near the edges. This is clearly not possible. Hence, we must have $c_{0} \geq 0$. Now, keeping an arbitrary non-negative $c_{0}$, one can in principle compute the saddle point energy, which is now parametrized by $c_{0} \geq 0$. So, one needs to minimize this energy further with respect to the parameter $c_{0}$ in the range $c_{0} \in[0, \infty)$. It turns out that this energy is an increasing function of $c_{0} \geq 0$ for all $-1<k<1$, and hence the minimum energy corresponds to $c_{0}=0$ even in this case $-1<k<1$. We do not show these calculations in detail here as they are a bit cumbersome. Hence, in the entire range $-1<p=k+1<2$, one finds that $c_{0}=0$ (in agreement with the physical argument above) and the full solution is just given by the particular solution

$$
d_{k}(z)=\frac{1}{J|k|} \frac{\sin \left(\frac{\pi p}{2}\right)}{\pi p}\left[z\left(\ell_{k}-z\right)\right]^{p / 2} .
$$

Going back to the $y$ coordinate and using $p=k+1$, the solution for all $-2<k<1$ can then be expressed as

$$
\tilde{\rho}_{k}(y)=\frac{1}{J|k|} \frac{\sin \left[\frac{\pi}{2}(k+1)\right]}{\pi(k+1)}\left(\frac{\ell_{k}^{2}}{4}-y^{2}\right)^{\frac{k+1}{2}}
$$

where $-\ell_{k} / 2<y<\ell_{k} / 2$. The support length $\ell_{k}$ is determined by the normalisation condition (62) and we get for all $-2<k<1$,

$$
\ell_{k}=\left(\frac{J|k| \pi(k+1)}{\sin \left[\frac{\pi}{2}(k+1)\right] B\left(\frac{k+3}{2}, \frac{k+3}{2}\right)}\right)^{\frac{1}{k+2}} .
$$

The solution in (64) can then be written in the customary scaling form $\tilde{\rho}_{k}(y)=\left(1 / \ell_{k}\right) F_{k}\left(y / \ell_{k}\right)$, where the scaling function $F_{k}(z)$ is given in Eq. (5) of the main text with the exponent $\gamma_{k}=(k+1) / 2$, as stated in the first line of Eq. (6) in the main text.

\section{SUMMARY OF THE AVERAGE DENSITY FOR LARGE $N$}

Let us finally summarize briefly our results for the leading large $N$ behavior of the average density for the three cases $-2<k<1, k>1$ and $k=1$. For both $-2<k<1$ and $k>1$, we find that the average density $\left\langle\rho_{N}(x)\right\rangle$ for large $N$ is independent of the inverse temperature $\beta$ and is described by the scaling form

$$
\left\langle\rho_{N}(x)\right\rangle \approx \frac{1}{\ell_{k} N^{\alpha_{k}}} F_{k}\left(\frac{x}{\ell_{k} N^{\alpha_{k}}}\right),
$$


where

$$
\alpha_{k}=\left\{\begin{array}{cc}
\frac{1}{k+2}, & -2<k<1 \\
\frac{k}{k+2}, & k>1,
\end{array}\right.
$$

and

$$
\ell_{k}=\left\{\begin{array}{lr}
\left(\frac{J|k| \pi(k+1)}{\sin \left[\frac{\pi}{2}(k+1)\right] B\left(\frac{k+3}{2}, \frac{k+3}{2}\right)}\right)^{\frac{1}{k+2}}, & -2<k<1 \\
\left(\frac{(2 J \zeta(k)(k+1))^{1 / k}}{B(1+1 / k, 1+1 / k)}\right)^{\frac{k}{k+2}}, & k>1 .
\end{array}\right.
$$

The scaling function $F_{k}(z)$, supported over $z \in[-1 / 2,1 / 2]$, is given explicitly by

$$
F_{k}(z)=\frac{1}{B\left(\gamma_{k}+1, \gamma_{k}+1\right)}\left(\frac{1}{4}-z^{2}\right)^{\gamma_{k}} ; \quad-1 / 2 \leq z \leq 1 / 2,
$$

where the exponent $\gamma_{k}$ is given by

$$
\gamma_{k}=\left\{\begin{array}{cc}
\frac{k+1}{2}, & -2<k<1 \\
\frac{1}{k}, & k>1 .
\end{array}\right.
$$

We find excellent agreement of our analytical prediction in (66) with numerical results, as shown in Fig. (3). The case $k=1$ is marginal and here the typical scale has logarithmic corrections. We find that the average density for large $N$ has the scaling form

$$
\left\langle\rho_{N}(x)\right\rangle \approx \frac{1}{\ell_{1} L_{N}} F_{1}\left(\frac{x}{\ell_{1} L_{N}}\right)
$$

where

$$
L_{N} \approx(N \ln N)^{1 / 3} ; \quad \text { and } \quad \ell_{1}=2(3 J)^{1 / 3} .
$$

The scaling function $F_{1}(z)$ is given explicitly by

$$
F_{1}(z)=6\left(\frac{1}{4}-z^{2}\right) ; \quad-1 / 2 \leq z \leq 1 / 2 .
$$

Let us end with a remark that the scaling function $F_{k}(z)$ in (69) (including the $k=1$ case) describing the large $N$ behavior of the average density is universal in the sense that it is independent of the inverse temperature $\beta$ and the coupling strength $J$-it only depends on the index $k$ that characterizes the exponent of the pairwise long-range power-law repulsive interaction between the particles. 


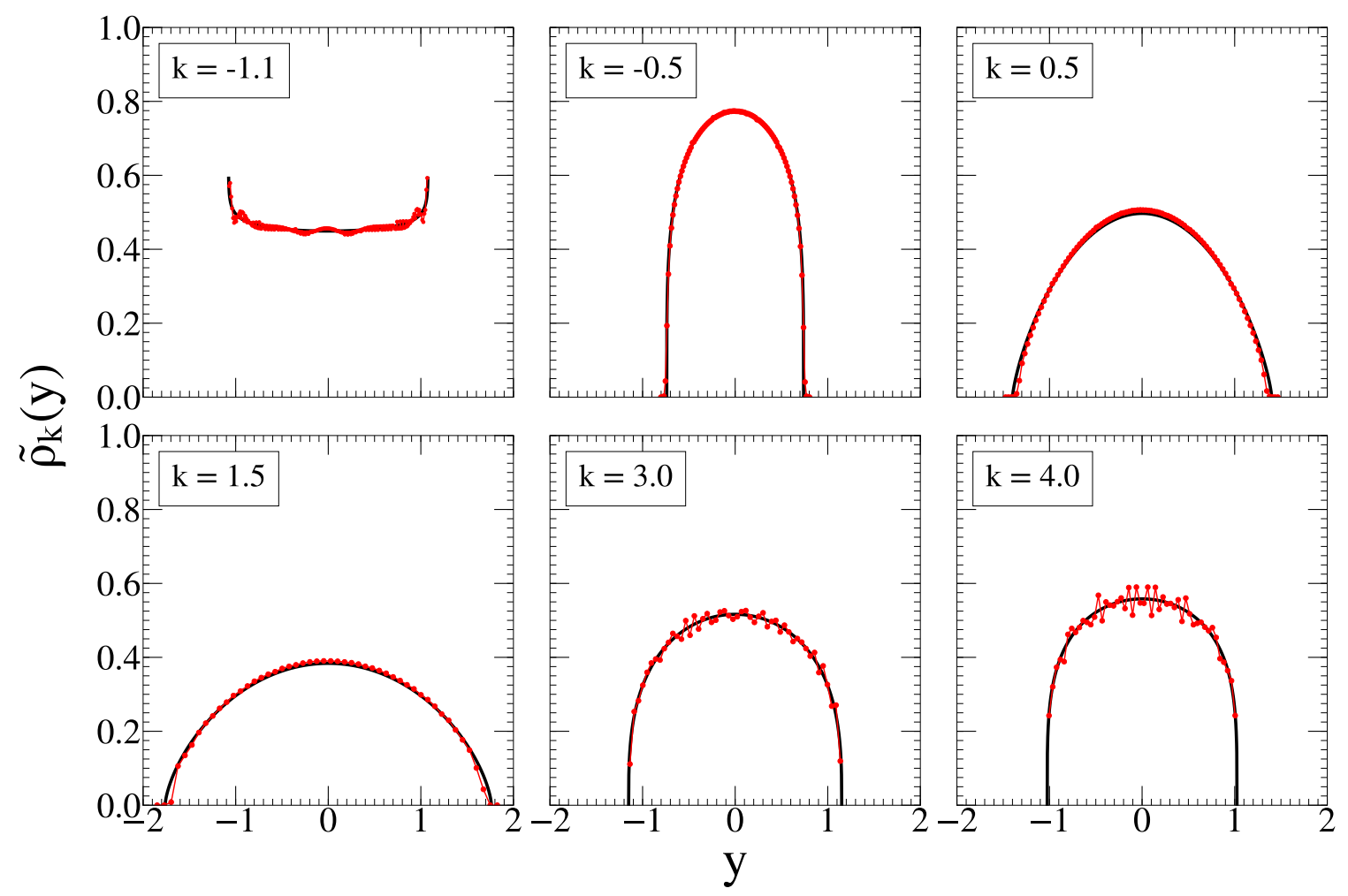

FIG. 3: The numerical (MC) average scaled density $\tilde{\rho}_{k}(y)$ vs $y$ (red dots) for different values of $k$ : for $-2<k<1$ in the top three panels and for $k>1$ in the bottom three panels (where $N=200$ and $\beta=2$ ). The numerical curves are compared to analytical predictions (black) in (66) with excellent agreement. Oscillations are somewhat prominent at higher $|k|$ due to the finite $N$ effects. The ensemble average is over $2 \times 10^{8} \mathrm{MC}$ samples.

1 D. S. Dean and S. N. Majumdar, Phys. Rev. E, 77, 041108 (2008).

2 R. Allez, J. -P. Bouchaud, S. N. Majumdar, P. Vivo, J. Phys. A.: Math. Theor. 46, 015001 (2012).

${ }^{3}$ F. G. Tricomi, Integral equations, Dover publications (1985).

4 S. N. Majumdar, G. Schehr, J. Stat. Mech. P01012 (2014).

${ }^{5}$ N. Ya Sonin, Studies of Cylinder functions and special polynomials (Gostekhizdat, moscow, 1954) (in Russian).

${ }^{6}$ G. Y. Popov, The elastic stress' concentration around dies, cuts, thin inclusions and reinforcements (1982).

7 H. Widom, J. Stat. Phys. 94, 347 (1999).

8 S. V. Buldyrev, M. Gitterman, S. Havlin, A. Ya. Kazakov, M. G. E. da Luz, E. P. Raposo, H. E. Stanley, G. M. Viswanathan, Physica A, 302, 148 (2001); PRE, 64, 041108 (2001).

9 A. Miron, arXiv: 1902.08974

10 B. Derrida, J. Stat. Mech. P07023 (2017).

11 J. Cividini, A. Kundu, A. Miron, D. Mukamel, J. Stat. Mech. P013203 (2017).

12 N. S. Landkof, Foundations of modern potential theory (Vol. 180), Springer (1972).

13 Note however that this homogeneous solution does have a physical meaning in the case where $h(z)=0$ but when the gas of particles is confined within a segment $[-R,+R]$. Indeed, in this case, and for $0<k<1$, the density is exactly given by this solution, see e.g. Ref. 12 p. 163. 